Corrigendum

\title{
Corrigendum to "Genotype-Phenotype Characterization of Novel Variants in Six Italian Patients with Familial Exudative Vitreoretinopathy"
}

\author{
Giancarlo Iarossi, ${ }^{1}$ Matteo Bertelli, ${ }^{2}$ Paolo Enrico Maltese, ${ }^{3}$ Elena Gusson, ${ }^{4}$ \\ Giorgio Marchini, ${ }^{4}$ Alice Bruson, ${ }^{3}$ Sabrina Benedetti, ${ }^{3}$ Sabrina Volpetti, ${ }^{5}$ Gino Catena, ${ }^{1}$ \\ Luca Buzzonetti, ${ }^{1}$ and Lucia Ziccardi ${ }^{6}$ \\ ${ }^{1}$ Department of Ophthalmology, Bambino Gesù IRCCS Children's Hospital, Rome, Italy \\ ${ }^{2}$ MAGI-Human Medical Genetics Institute, Bolzano, Italy \\ ${ }^{3}$ MAGI-Human Medical Genetics Institute, Rovereto, Italy \\ ${ }^{4}$ Eye Clinic, Department of Neurosciences, Biomedicine and Movement, University and AOUI (Azienda Ospedaliera \\ Universitaria Integrata) of Verona, Verona, Italy \\ ${ }^{5}$ Dipartimento Anestesia e Rianimazione Materno Infantile, Ospedale San Filippo Neri, Rome, Italy \\ "G.B. Bietti" Foundation, IRCCS, Rome, Italy
}

Correspondence should be addressed to Paolo Enrico Maltese; paolo.maltese@assomagi.org

Received 14 September 2017; Accepted 4 October 2017; Published 30 November 2017

Copyright @ 2017 Giancarlo Iarossi et al. This is an open access article distributed under the Creative Commons Attribution License, which permits unrestricted use, distribution, and reproduction in any medium, provided the original work is properly cited.

In the article titled "Genotype-Phenotype Characterization of Novel Variants in Six Italian Patients with Familial Exudative Vitreoretinopathy" [1], there was an error in the variation in the NDP gene c.313G>C. Accordingly, "p.(Ala105Phe)" should be corrected to "p.(Ala105Pro)" in the Results and Discussion. Also, Table 1 and Figure 5 should be corrected as follows: 
TABLE 1: Features of genetic variations found in FEVR families.

\begin{tabular}{|c|c|c|c|c|c|c|c|c|}
\hline $\begin{array}{l}\text { Family ID } \\
\text { Gene }\end{array}$ & Genotype & Nucleotide change & Amino acid change & SIFT & Polyphen & Mutation taster & Classification & References \\
\hline $\begin{array}{l}\text { Fam. 1 } \\
\text { FZD4 } \\
\text { NM_012193 }\end{array}$ & Het & c. $277 \mathrm{C}>\mathrm{T}$ & p. $\left(\mathrm{G} \ln 93^{*}\right)$ & - & - & - & Pathogenic & Novel variant \\
\hline $\begin{array}{l}\text { Fam. } 2 \\
\text { FZD4 } \\
\text { NM_012193 }\end{array}$ & Het & c. $542 \mathrm{G}>\mathrm{A}$ & p.(Cys181Tyr) & $\mathrm{T}$ & $\operatorname{PrD}$ & $\mathrm{DC}$ & Pathogenic & {$[31]$} \\
\hline $\begin{array}{l}\text { Fam. } 3 \\
\text { FZD4 } \\
\text { NM_012193 }\end{array}$ & Het & c. $611 \mathrm{G}>\mathrm{T}$ & p.(Cys204Phe) & $\mathrm{D}$ & $\operatorname{PrD}$ & DC & Likely pathogenic & Novel variant \\
\hline $\begin{array}{l}\text { Fam. } 4 \\
\text { NDP } \\
\text { NM_000266 }\end{array}$ & Hemi & c. $362 \mathrm{G}>\mathrm{A}$ & p. $(\operatorname{Arg} 121 \mathrm{Gln})$ & $\mathrm{D}$ & $\operatorname{PrD}$ & DC & Pathogenic & {$[25]$} \\
\hline $\begin{array}{l}\text { Fam. } 5 \\
N D P \\
\text { NM_000266 }\end{array}$ & Hemi & c. $313 \mathrm{G}>\mathrm{C}$ & p.(Ala105Pro) & $\mathrm{D}$ & $\operatorname{PrD}$ & DC & Likely pathogenic & Novel variant \\
\hline $\begin{array}{l}\text { Fam. } 6 \\
\text { TSPAN12 } \\
\text { NM_012338 }\end{array}$ & Het & c. $67-2 A>G$ & Defective splicing & - & - & - & Likely pathogenic & Novel variant \\
\hline
\end{tabular}
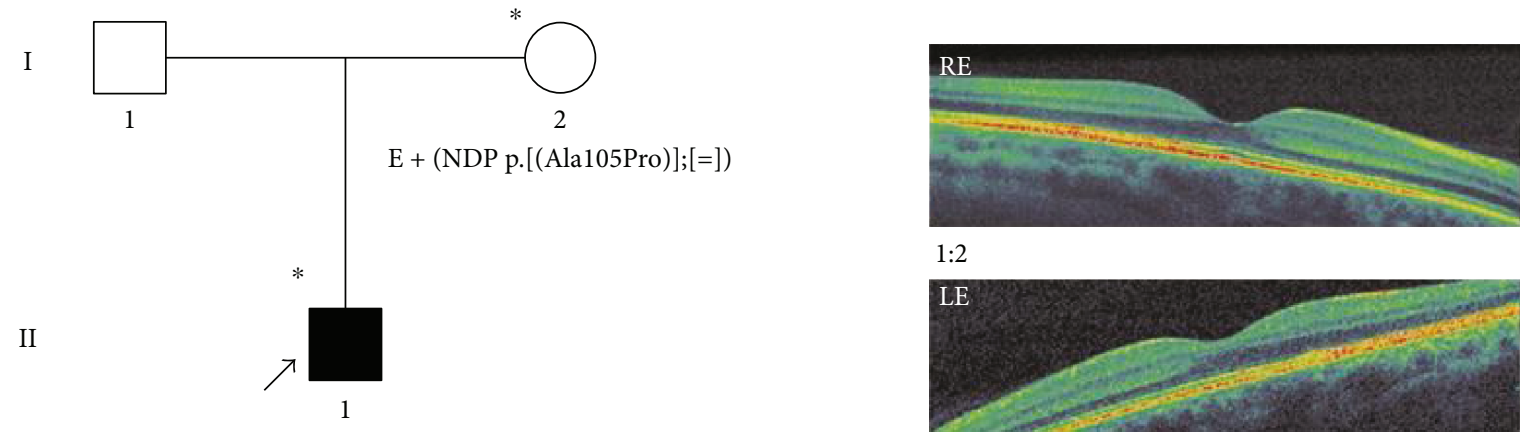

$1: 2$

$\mathrm{E}+(\mathrm{NDP} p \cdot[($ Ala105Pro) $])$
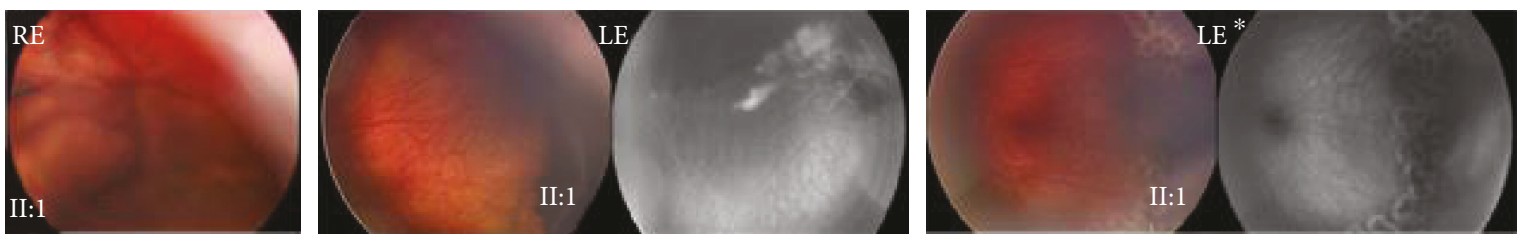

Figure 5: Pedigree and ocular features of family 5. Color fundus photographs by RetCam from the 3 y/o male proband (II:1) of family 5 showing a closed funnel total retinal detachment in the right eye and peripheral exudation with vascular abnormalities before and after laser treatment in the left eye. Fluorescein angiograms performed with RetCam before and after laser treatment showing peripheral ischemic areas with leakage mainly in temporal and inferior peripheral retina in the left eye. Top right, OCT macular scans from the proband's mother (I:2) showing normal retinal features. * Documented clinical evaluation; E+, positive to genetic test; RE, right eye; $\mathrm{LE}$, left eye; $\mathrm{LE}^{*}$, images taken at a subsequent examination after the laser treatment.

\section{References}

[1] G. Iarossi, M. Bertelli, P. E. Maltese et al., "Genotype-phenotype characterization of novel variants in six Italian patients with familial exudative vitreoretinopathy," Journal of Ophthalmology, vol. 2017, Article ID 3080245, 10 pages, 2017. 


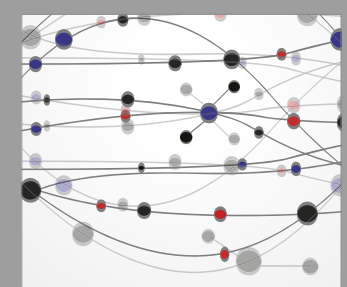

The Scientific World Journal
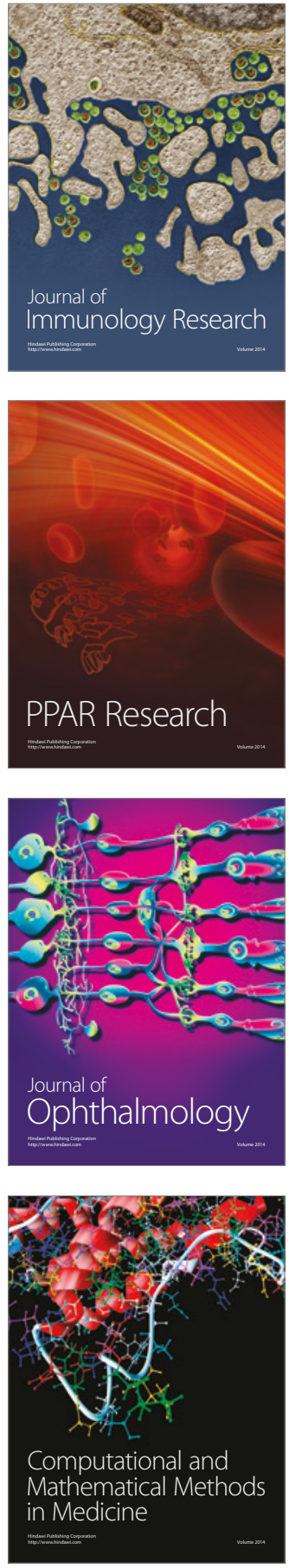

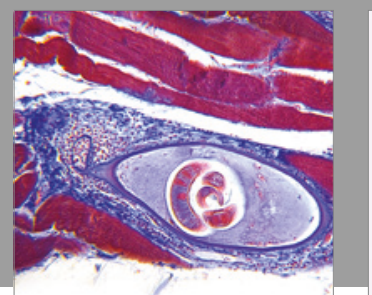

Gastroenterology Research and Practice
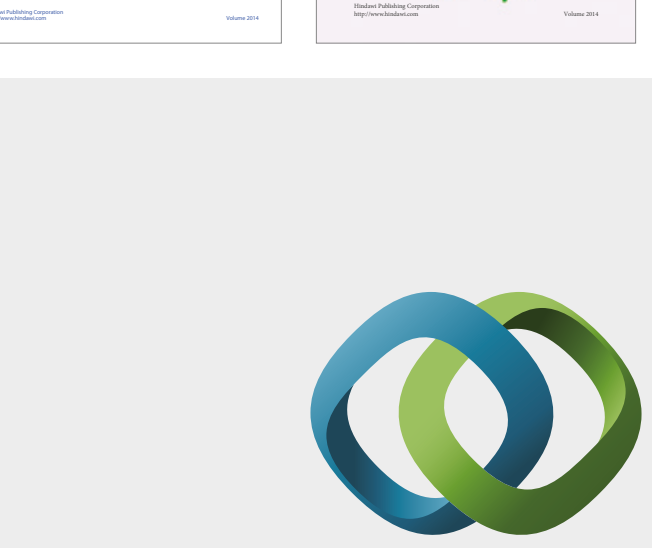

\section{Hindawi}

Submit your manuscripts at

https://www.hindawi.com
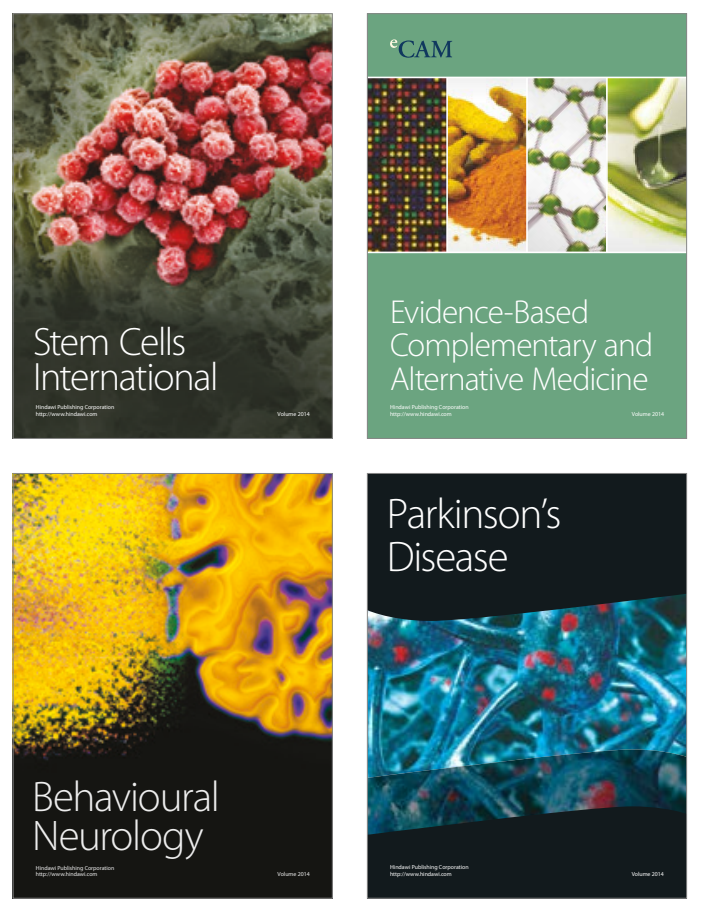
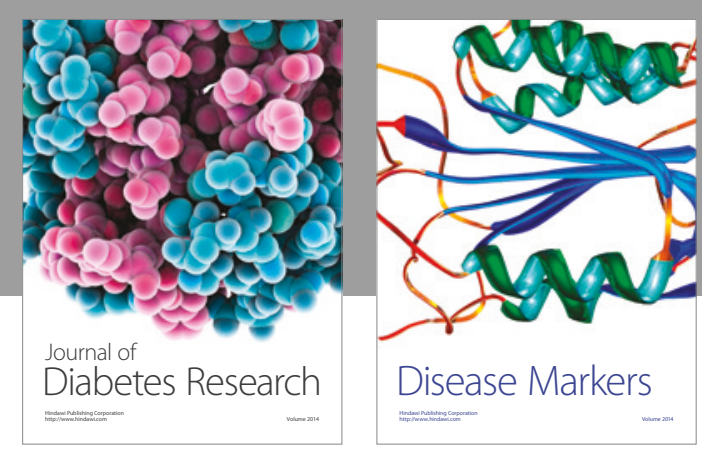

Disease Markers
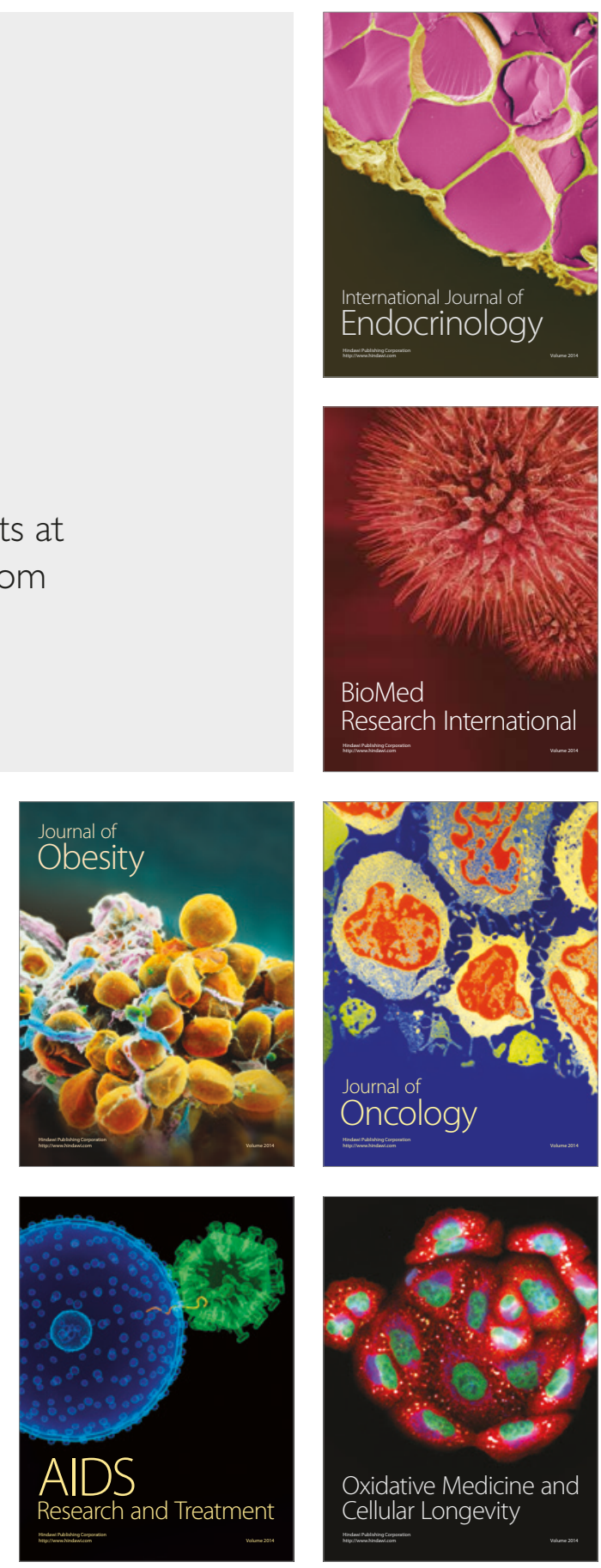